\title{
Two-Port Networks to Model Galvanic Coupling for Intrabody Communications and Power Transfer to Implants
}

\author{
Laura Becerra-Fajardo \\ Department of Information and \\ Communications Technologies \\ Universitat Pompeu Fabra \\ Barcelona, Spain \\ laura.becerra@upf.edu
}

\author{
Marc Tudela-Pi \\ Department of Information and \\ Communications Technologies \\ Universitat Pompeu Fabra \\ Barcelona, Spain \\ marc.tudela@upf.edu
}

\author{
Antoni Ivorra \\ Serra Húnter Fellow \\ Department of Information and \\ Communications Technologies \\ Universitat Pompeu Fabra \\ Barcelona, Spain \\ antoni.ivorra@upf.edu
}

\begin{abstract}
Galvanic coupling, or more precisely, volume conduction, can be used to communicate with and to transfer power to electronic implants. Since no bulky components for power, such as coils or batteries, are required within the implants, this strategy can yield very thin devices suitable for implantation by injection. To design the circuitry of both the implants and the external systems, it is desirable to possess a model that encompasses the behavior of these circuits and also the volume conduction phenomenon. Here we propose to model volume conduction with a two-port network so that the whole system can be studied in circuit simulators. The two-port network consists only of three impedances whose values can be obtained through simple measurements or through numerical methods. We report a validation of this modeling approach in a geometrically simple in vitro setup that allowed us to determine the impedances of the two-port network not only by performing measurements or through a finite element method study but also through an analytical solution.
\end{abstract}

Keywords - galvanic coupling, volume conduction, neuroprosthetics, implants, power transfer, SPICE

\section{INTRODUCTION}

We are working on the development of injectable microstimulators that avoid the need to embed bulky components for power such as coils and batteries [1]. We envision very thin $(<1 \mathrm{~mm})$ implants with digital communications, and stimulation and sensing capabilities. In [2], we in vivo demonstrated proof-of-concept prototypes capable of selective stimulation.

The stimulation implants we are developing act as rectifiers of innocuous high frequency current bursts $(\geq 1 \mathrm{MHz}$ ) that are injected by an external generator into the tissues through superficial electrodes. In essence, we are proposing the use of galvanic coupling, or more precisely, volume conduction, for power transfer and communications.

Remarkably, although volume conduction for intrabody communications has been proposed lately by different research groups [3], its use for powering implants has been neglected. We conjecture that reluctance to use volume conduction for power transfer may arise from not recognizing two facts. First, large magnitude high frequency (> $1 \mathrm{MHz}$ ) currents can safely flow through the human body if applied as short bursts. Second, to obtain a sufficient voltage drop across its two intake (pick-up) electrodes, the implant can be shaped as a thin and flexible elongated body suitable for minimally invasive percutaneous deployment (Fig. 1). In [4] we have shown that it should be possible to safely supply powers well above $1 \mathrm{~mW}$ to thin elongated implants (diameter $\leq 1 \mathrm{~mm}$, length $\geq 20 \mathrm{~mm}$ ) by means of volume conduction. Such power transfer level is comparable to those obtained by other wireless power transfer strategies for implantable systems [5].

In order to design the circuitry of both the implants and the external systems, it is desirable to possess a model that encompasses the behavior of these circuits and also the phenomenon of volume conduction through the living tissues and the electrodes. Such complete model will enable testing different features of the circuits before implementing and testing them in vivo. Here we propose to model volume conduction with a two-port network so that the whole system can be studied in circuit simulators (Fig. 2). The proposed two-port network consists in a T-equivalent circuit with three impedances.

Two-port networks were proposed in the past for modeling galvanic coupling [6]. However, the proposed model consisted of six impedances instead of only three impedances and it was not described how those impedances could be obtained.

The two external electrodes, the tissues and the two implant electrodes form a two-port network in which the ports voltages and currents are linearly related. In particular, since tissues and electrodes are passive and linear for low current densities, they form a reciprocal network [7]. This means that the voltages and currents can be expressed as:

$$
\left[\begin{array}{l}
v_{1} \\
v_{2}
\end{array}\right]=\left[\begin{array}{ll}
z_{11} & z_{12} \\
z_{12} & z_{22}
\end{array}\right]\left[\begin{array}{l}
i_{1} \\
i_{2}
\end{array}\right]
$$

where $v_{1}$ is the voltage across the two external electrodes, $v_{2}$ is the voltage across the two implant electrodes, $i_{1}$ is the current through the two external electrodes, $i_{2}$ is the current

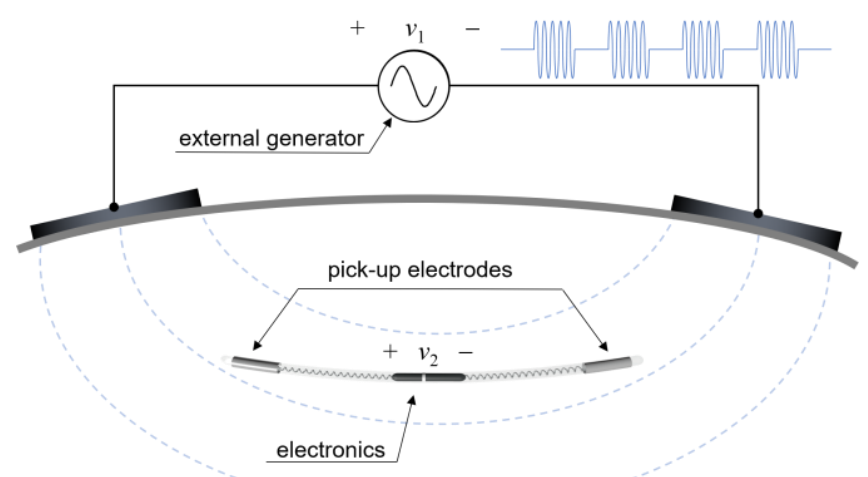

Fig. 1. Schematic representation of an implant powered by galvanic coupling (not to scale). 




Fig. 2. Proposed two-port model representing the coupling between the two electrodes of the external generator and the two electrodes of the implant. It consists of three impedances.

through the two implant electrodes and $z_{x y}$ are the impedance parameters. It can be readily demonstrated that the proposed T-equivalent circuit shown in Fig. 2 agrees with the system of equations in (1).

By definition:

$$
\left.z_{11} \stackrel{\text { def }}{=} \frac{v_{1}}{i_{1}}\right|_{i_{2}=0},\left.z_{22} \stackrel{\text { def }}{=} \frac{v_{2}}{i_{2}}\right|_{i_{1}=0},\left.z_{12} \stackrel{\text { def }}{=} \frac{v_{1}}{i_{2}}\right|_{i_{1}=0}=\left.\frac{v_{2}}{i_{1}}\right|_{i_{2}=0}
$$

The above equations (2) indicate that the impedance parameters can be found by injecting known currents and measuring the resulting voltages across the external electrodes and across the implant electrodes. In the study presented here it is illustrated how this can be done by means of an analytical solution, by performing a finite element method study, or by performing actual measurements.

\section{METHODS}

\section{A. Determination of network impedances}

The impedance parameters for the two-port network model were determined using three different methods: through an analytical solution, through a finite element method study, and by performing actual measurements in an in vitro setup. The three methods were used to model the scenario shown in Fig. 3, in which two parallel metallic plates (cross-sectional area $A=2600 \mathrm{~mm}^{2}$ ) acting as external electrodes are separated by a saline medium with a measured conductivity of $0.592 \mathrm{~S} / \mathrm{m}$, and two metallic spheres (diameter, $D=1 \mathrm{~mm}$ ) acting as implant electrodes are immersed in it.

1) Analytical solution: As defined in (2), $z_{11}$ corresponds to the impedance between the two external electrodes. Considering the model presented in Fig. 3, $z_{11}$ can be calculated as:

$$
z_{11}=\frac{\ell_{1}}{\sigma A}
$$

where $\ell_{1}$ is the separation distance between the external electrodes, and $\sigma$ is the tissue conductivity.

Similarly, it can be noticed that $z_{22}$ has been defined as the impedance between the implant electrodes. In this study, these electrodes are modeled as conductive spheres with a separation distance $\ell_{2}$, much larger than its diameter $D$. Assuming that the dimensions of the medium where the implant is located are much larger than the size of the implant (i.e. $\ell_{1} \gg \ell_{2}$, and $A \gg D$ ), then according to [8]:

$$
z_{22}=\frac{1}{\sigma \pi D}
$$

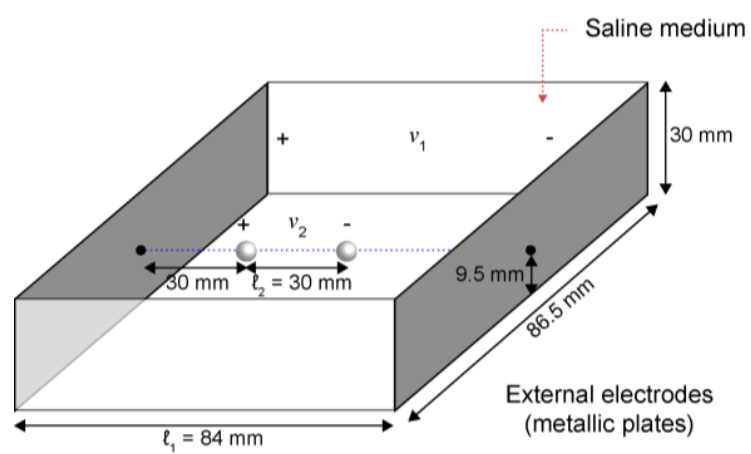

Fig. 3. Modeled scenario. Two metallic plates act as external electrodes, and two metallic spheres $(D=1 \mathrm{~mm})$ act as implant electrodes.

The applied electric field $E$, can be calculated using the voltage across the two external electrodes $v_{1}$ and the distance $\ell_{1}$ between the two electrodes:

$$
E=\frac{v_{1}}{\ell_{1}}
$$

Having in mind the previous considerations, when $i_{2}=0$ :

$$
v_{2}=E \ell_{2} \cos \theta
$$

where $\theta$ is the angle between the applied electric field $E$ and the axis formed by the implant electrodes. For the particular case of the modeled scenario (Fig. 3), this angle is $0^{\circ}$.

$v_{2}$ can be also determined by analyzing the two-port model (Fig.2), for a null $i_{2}$ :

$$
v_{2}=v_{1} \frac{z_{12}}{z_{11}}
$$

Then, substituting (3, 5, and 6) into (7) the analytic value of $z_{12}$ can be determined:

$$
\begin{gathered}
E \ell_{2} \cos \theta=E \ell_{1} \frac{z_{12} \sigma A}{\ell_{1}} \\
z_{12}=\frac{\ell_{2}}{\sigma A} \cos \theta
\end{gathered}
$$

Finally, since $z_{12}=z_{21}$, replacing (3, 4, and 9) into (1) the analytical relation of this two-port system is expressed as:

$$
\left[\begin{array}{l}
v_{1} \\
v_{2}
\end{array}\right]=\left[\begin{array}{cc}
\frac{\ell_{1}}{\sigma A} & \frac{\ell_{2}}{\sigma A} \cos \theta \\
\frac{\ell_{2}}{\sigma A} \cos \theta & \frac{1}{\sigma \pi D}
\end{array}\right]\left[\begin{array}{l}
i_{1} \\
i_{2}
\end{array}\right]
$$

2) Finite element method (FEM) study: This study was performed with a FEM software platform (COMSOL Multiphysics 4.4) using the "Electric Currents" application mode. The geometrical model consists of two metallic plates (length $=86.5 \mathrm{~mm}$, height $=30 \mathrm{~mm}$, width $=1 \mathrm{~mm}$, conductivity $=1 \times 10^{5} \mathrm{~S} / \mathrm{m}$, relative permittivity = 1) that modelled the external electrodes. These plates were separated $84 \mathrm{~mm}\left(\ell_{1}\right)$ by a saline medium (conductivity = $0.592 \mathrm{~S} / \mathrm{m}$, relative permittivity $=80$ ), as shown in Fig. 3 . The implant electrodes were modeled using two metallic spheres $\left(D=1 \mathrm{~mm}\right.$, conductivity $=1 \times 10^{5} \mathrm{~S} / \mathrm{m}$, relative permittivity $=1)$ separated $30 \mathrm{~mm}\left(\ell_{2}\right)$ and located between the metallic plates, at a distance of $30 \mathrm{~mm}$ from one metallic plate (Fig. 3). The software automatically generated a mesh of 249914 tetrahedral elements.

The $z_{11}$ and $z_{12}$ impedance parameters were found by simulating the injection of a reference electric current with 
the external electrodes, and by measuring the voltages across the external electrodes and the implant electrodes. A similar procedure was used to find the $z_{22}$ and $z_{21}$ impedance parameters: a reference current was injected with the implant electrodes, and the voltages across the external electrodes and the implant electrodes were measured.

3) Actual measurements: The in vitro setup represented in Fig. 3 was implemented and was used to perform actual measurements of the impedance parameters. High frequency bursts $(f=1 \mathrm{MHz}, \mathrm{T}=500 \mu \mathrm{s})$ were delivered across the external electrodes by means of a function generator (BK4064 by BK Precision) followed by a high voltage amplifier (WMA 300 by Falco Systems). The voltages across the external electrodes and the implant electrodes, as well as the currents flowing through the two pairs of electrodes were measured using a digital oscilloscope (TPS2014 by Tektronix, Inc). Afterwards, the impedances $\mathrm{z}_{\mathrm{xy}}$ were calculated as in (2).

A conductivity meter (HI98312 DIST 6 by Hanna Instruments) was used to measure the conductivity of the saline medium. This conductivity matches that used for the analytical solution and the FEM study.

\section{B. Use of the obtained two-port network in a non-linear problem}

Three SPICE transient simulations (solved in LTspice XVII by Linear Technology Corp.) were performed of the circuit shown in Fig. 4A which is intended to reproduce the setup in Fig. 4B. The values for the impedances of the twoport model $z_{11}-z_{12}, z_{12}$ and $z_{22}-z_{12}$ were defined using the results obtained with the three types of impedance parameters measurements described above. For simplicity, the implant electronics consisted of a single Schottky diode (RB521ZS-30 by RHOM Semiconductor). (This diode is used in the charge-balance rectifier described in [1].) The external generator was set to an amplitude of $8.4 \mathrm{~V}_{\mathrm{RMS}}$. It delivered 1 burst of $500 \mu \mathrm{s}$ duration at a frequency $f$ of $1 \mathrm{MHz}$.

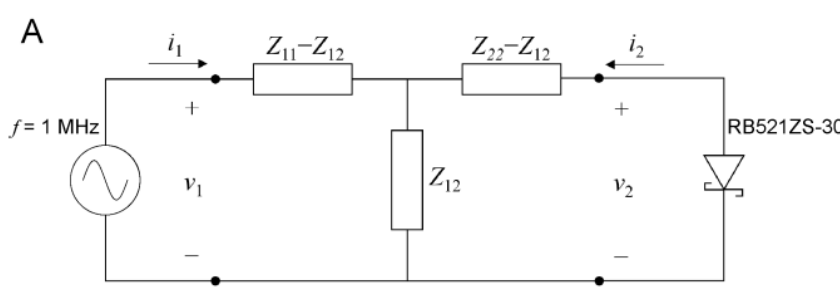

B

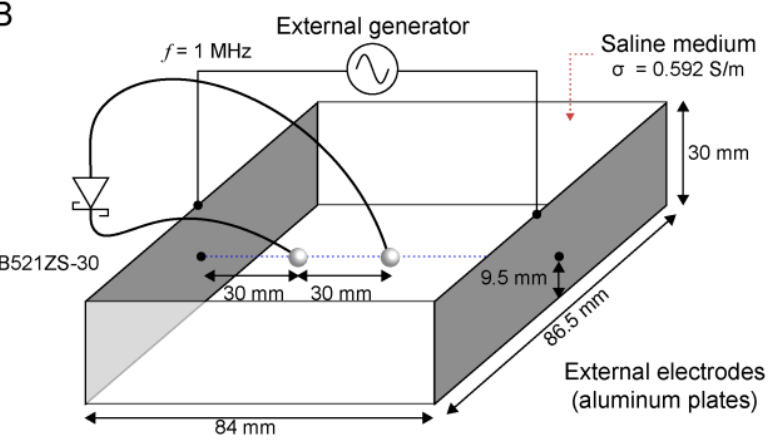

Fig. 4. A) Circuit for SPICE simulation, in which the T-equivalent is connected to an ac generator and a Schottky diode. B) Scenario modeled by the above circuit.
The voltages across the Schottky diode obtained for the three simulations were then compared to the voltage measured across a RB521ZS-30 Schottky diode connected to the spheres in the in vitro setup shown in Fig. 4B. This was done using a digital oscilloscope (TPS2014 by Tektronix, Inc).

\section{RESULTS}

Table I shows the impedance parameters obtained with the three methods described above (analytical solution, FEM study, actual measurements). To calculate the relative differences of the impedance parameters found with the FEM study and the actual measurements, the analytical results were used as reference values. The maximum relative difference of the FEM study results, which corresponds to the relative difference obtained for $z_{22}$, is $0.53 \%$. In the case of the actual measurements obtained in vitro, the maximum relative difference was $12.5 \%$, which corresponds to the $z_{12}$ impedance parameter.

In the case of the three impedances for the T-equivalent that represent the coupling between the two electrodes of the generator and the two electrodes of the implant in the twoport model (Fig. 2), the maximum relative difference is $5 \%$. This difference is obtained for impedance $z_{22}-z_{12}$ with the actual measurements in vitro method.

The SPICE simulations of the two-port network connected to the Schottky diode's SPICE model (Fig. 4A) and the generator were compared to the real measurements obtained with a Schottky diode connected to the two spherical electrodes (Fig. 4B). The voltage across the diode during the first $5 \mu \mathrm{s}$ of the first high frequency burst obtained with the real measurement in vitro and with the three SPICE simulations are shown in Fig. 5.

TABLE I. OBTAINED $Z_{\mathrm{XY}}$ PARAMETERS $(\Omega)$

\begin{tabular}{lcccc}
\hline \hline Method & $\mathrm{Z}_{11}$ & $\mathrm{Z}_{12}$ & $\mathrm{Z}_{21}$ & $\mathrm{Z}_{22}$ \\
\hline Analytical & 54.335 & 19.405 & 19.405 & 537.686 \\
FEM & 54.334 & 19.405 & 19.405 & 540.539 \\
In vitro & 56.452 & 21.831 & 20.336 & 512.769 \\
\hline \hline
\end{tabular}

In Fig. 5 it can be observed that the voltage across the Schottky diode in the real measurement is very similar to those simulated in SPICE using the impedance parameters of the two-port model obtained with the analytical solution, the FEM study, and the actual measurements in vitro.

\section{DISCUSSION AND CONCLUSIONS}

In here we have proposed a two-port network consisting of three impedances for modelling volume conduction. The network represents the coupling between two electrodes of an external system and two electrodes of an implant. The two-port network can be easily integrated into a SPICE simulator to simulate the behavior of any implant electronics, including the very thin implants we envision for wireless stimulation and sensing. The waveforms obtained with these simulations would allow us to predict the behavior of the implant in terms of power transfer and intrabody communications (e.g. study of waveform distortion). 


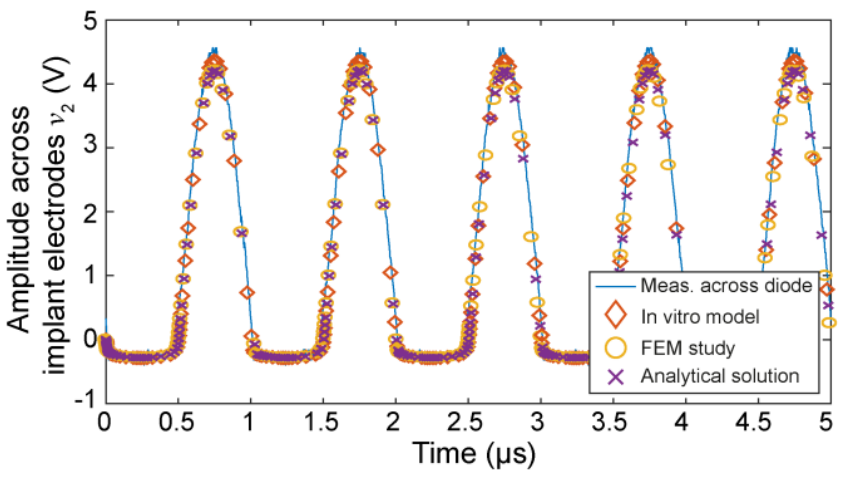

Fig. 5. Voltage measured across the Schottky diode during the first $5 \mu$ s of a high frequency burst delivered by the external generator. The continuous line represents the voltage across the Schottky diode in the in vitro setup shown in Fig 4 . The three symbols $(\diamond$, o and $\times)$ represent the results obtained for the SPICE simulations using the obtained port models.

For illustrative purposes, the scenario modeled here was very simple and the reactance of the impedances was negligible. That is, the impedances were in fact resistances. However, it must be noted that the applied methodology is also valid for complex impedances as those that would be observed across living tissues and also across electrodes when measured at lower frequencies.

The small relative differences of the impedance parameters obtained with the FEM study and the actual measurements in vitro compared to those obtained with the analytical solution validate the results of each of them. These differences are most likely due to geometrical tolerances in the construction of the in vitro setup.

The real measurements with a Schottky diode connected to two spheres which were located in the saline medium of the in vitro setup were very similar to the results obtained with the SPICE simulations. This confirms the validity of the modeling approach.

The modeling approach used here can also be applied to scenarios where more than one implant is present. In those cases, more ports would be added to the network. Equation (11) and Fig. 6 illustrate the system of equations and the T-equivalent circuit for a three-port port reciprocal network. This could be used, for instance, to study whether it is possible to power two implants that are closely located.

$$
\left[\begin{array}{l}
v_{1} \\
v_{2} \\
v_{3}
\end{array}\right]=\left[\begin{array}{lll}
z_{11} & z_{12} & z_{12} \\
z_{12} & z_{22} & z_{23} \\
z_{12} & z_{23} & z_{33}
\end{array}\right]\left[\begin{array}{l}
i_{1} \\
i_{2} \\
i_{3}
\end{array}\right]
$$

One drawback of using galvanic coupling for power transfer to implants is its very poor energy efficiency. However, it must be noted that we have shown in the past that it will be possible to use existing rechargeable portable batteries (> $100 \mathrm{Wh} / \mathrm{kg}$ ) to power the external generator that delivers the high frequency currents to drive implantable electronic devices, even in a highly demanding scenario [9]. Therefore, we anticipate it will be feasible to develop portable external systems to be carried easily by patients. The proposed two-port network for modelling galvanic coupling is not only helpful for designing implant electronics, but also for designing this portable external system, and to evaluate its performance for powering implants and for communications.

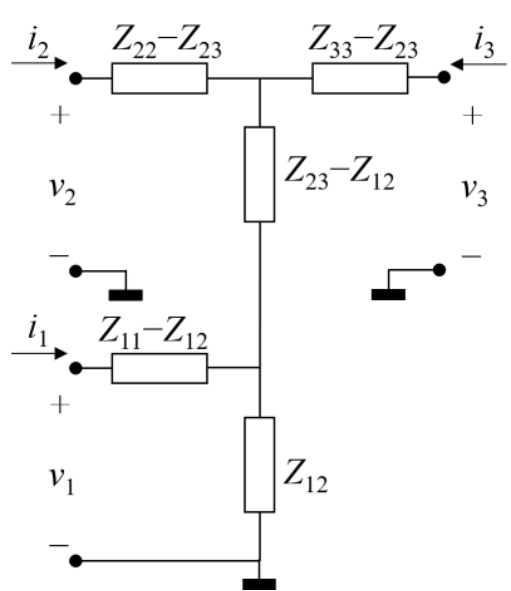

Fig. 6. T-equivalent circuit for a three-port reciprocal network.

\section{ACKNOWLEDGMENT}

This project has received funding from the European Research Council (ERC) under the European Union's Horizon 2020 research and innovation programme (grant agreement No 724244). The authors would like to express their gratitude to Aracelys Garcia for her help in implementing the in vitro setup.

\section{REFERENCES}

[1] A. Ivorra, L. Becerra-Fajardo, and Q. Castellví, "In vivo demonstration of injectable microstimulators based on chargebalanced rectification of epidermically applied currents," Journal of Neural Engineering, vol. 12(6), pp. 066010, 2015.

[2] L. Becerra-Fajardo, M. Schmidbauer and A. Ivorra, "Demonstration of $2 \mathrm{~mm}$ thick microcontrolled injectable stimulators based on rectification of high frequency current bursts," IEEE Trans Neural Syst Rehabil Eng, vol. 25(8), pp. 1343-1352, 2017.

[3] M. Seyedi, B. Kibret, D.T. Lai, and M. Faulkner, "A survey on intrabody communications for body area network applications," IEEE Trans Biomed Eng, vol. 60(8), pp. 2067-2079, 2013.

[4] M. Tudela-Pi, L. Becerra-Fajardo, and A. Ivorra, "Powering Implants by Galvanic Coupling: A Validated Analytical Model Predicts Powers Above $1 \mathrm{~mW}$ in Injectable Implants," IFMBE Proceedings, vol. 68(3), pp.23-26, 2018.

[5] K. Agarwal, R. Jegadeesan, Y.-X. Guo, and N. V. Thakor, "Wireless Power Transfer Strategies for Implantable Bioelectronics," IEEE Rev. Biomed. Eng., vol. 10, pp. 136-161, 2017.

[6] M.S. Wegmueller, M. Oberle, N. Felber, N. Kuster, and W. Fichtner, "Signal Transmission by Galvanic Coupling Through the Human Body," IEEE Trans Instrum Meas, vol. 59(4), pp. 963-969, 2009.

[7] W.H. Hayt, J. Kemmerly, and S.M. Durbin, "Two-port networks," in Engineering Circuit Analysis, Eight Edition, New York: McGrawHill, pp. 687-732, 2012.

[8] Grimnes, S. and Martinsen, Ø.G., Bioimpedance \& Bioelectricity Basics, 2nd ed. Elseiver, Oxford 2008. pp. 161-204.

[9] L. Becerra-Fajardo, R. Garcia-Arnau, and A. Ivorra, "Injectable Stimulators Based on Rectification of High Frequency Current Bursts: Power Efficiency of $2 \mathrm{~mm}$ Thick Prototypes - Converging Clinical and Engineering Research on Neurorehabilitation II," 2017, pp. 667671. 